\title{
Relationship between psychological state and level of activity of extrinsic gut innervation in patients with a functional gut disorder
}

\author{
A V Emmanuel, H J Mason, M A Kamm
}

\begin{abstract}
Background-Anxiety and depression are known to be associated with alterations in central autonomic activity, and this may manifest as a functional gut disturbance. However, the final expression of motility disturbance is non-specific and nonquantifiable. This study examines the relationship between psychological state and psychosocial functioning with a new direct measure of the level of activity of extrinsic autonomic gut innervation, rectal mucosal Doppler blood flow.

Materials and methods-Thirty four female patients (mean age 36 years, range 19-45) with constipation for greater than five years and 19 healthy women (mean age 38 years, range 21-60) were studied. They completed the general health questionnaire-28 point scale (GHQ-28; psychosocial functioning) and the Bem sex role inventory (BSRI; an index of women's psychological feelings about their own femininity). On the same day they underwent measurement of rectal mucosal Doppler blood flow, a new validated measure of the activity of gut extrinsic nerve innervation. Measurements were made during the follicular phase and in the fasted state.
\end{abstract}

Results-Women with constipation scored higher on the total GHQ-28 score and the somatisation $(p=0.05)$ and anxiety $(p=0.05)$ subscales of the GHQ-28. There was a negative correlation between mucosal blood flow and GHQ somatisation subscale $\quad(r=-0.45, \quad \mathrm{p}<0.005)$, anxiety $(r=-0.38, \quad \mathrm{p}<0.05), \quad$ and depression $(r=-0.40, p<0.01)$ scores in women with constipation. Although constipated women scored no higher than controls on the BSRI, there was a significant negative correlation between blood flow and BSRI score $(r=-0.49, p<0.005)$ for constipated women.

Conclusions-General psychosocial function, somatisation, anxiety, depression, and feelings about female role are impaired in women with constipation and associated with altered rectal mucosal blood flow, a measure of extrinsic gut innervation. These findings suggest that psychological factors are likely to influence gut function via autonomic efferent neural pathways.

(Gut 2001;49:209-213)

Keywords: gut function; gut innervation; constipation; psychological state
Specific alterations of gastrointestinal motility can be produced in animals by stimulating different regions of the brain. ${ }^{12}$ In certain circumstances the neurotransmitter responsible for these changes has been identified, particularly in relation to changes in colonic motility. ${ }^{3-5}$ For example, intracerebroventricular injection of corticotrophin releasing hormone in rats, a peptide found predominantly in the hypothalamus and other brain areas associated with arousal and autonomic activity, produces changes in colonic motility. The same neurotransmitter mediates the colonic motility response to acute stress. ${ }^{6}$ In humans, alterations in the level of arousal, or feelings of anxiety and depression, are associated with alterations of brainstem and cortical autonomic activity. ${ }^{78}$ It seems likely therefore that changes in cerebral activity which influence gut behaviour are mediated via extrinsic autonomic pathways.

In humans there is a well recognised association between emotional and psychological factors and gut function. Early studies included the observations on a soldier with a gastrocutaneous fistula following a gun shot injury ${ }^{9}$ and on "Tom's stomach", ${ }^{10}$ in which changes in gastric motility, mucosal erythema, and secretion were observed in response to acute emotional changes. Similar changes in the large bowel were observed in medical students who showed alterations in rectal erythema when acute emotional distress was induced. ${ }^{11}$

These studies in animals and humans have established a direct link between acute emotional changes and control of gut function. Evidence for chronic changes in psychological state and gut function have been more difficult to establish, and have relied more on psychometric testing in groups of patients with known functional disorders. A direct physiological link has not been established in these patients. Indirect physiological evidence however comes from cardiovascular autonomic testing in patients with functional gut disorders. ${ }^{12}{ }^{13}$ The aim of this study was to utilise a tool which has been developed to allow direct measurement of the level of activity of the autonomic innervation to the gut, and establish whether gut extrinsic autonomic activity relates to quantitated measures of psychological morbidity.

To investigate this relationship patients with idiopathic constipation were studied. Previous

Abbreviations used in this paper: GHQ-28, general health questionnaire -28 point scale; BSRI, Bem sex role inventory; IIP-I, inventory of interpersonal problems-intimacy subscale. 
reports on these patients have shown both evidence of psychological morbidity ${ }^{14-17}$ and clearly demonstrable physiological changes such as slowing in whole gut transit. Constipated patients, whether with slow or normal whole gut transit, tend to have higher scores on scales of somatisation, interpersonal sensitivity and hostility, paranoid ideation, and anxiety and depression. ${ }^{14-17} \mathrm{~A}$ general measure of psychosocial functioning, the general health questionnaire-28 point scale (GHQ-28), ${ }^{18}$ was employed to assess these features. The GHQ-28 has been validated against structured psychiatric interview schedules and been found to be an effective, sensitive, and specific instrument in detecting occult mental dysfunction in both patients with organic ${ }^{19}$ and non-organic illness. $^{20}$

Most of these patients are women, ${ }^{21}$ and a history of a traumatic childhood ${ }^{14}$ or repeated physical or sexual abuse $e^{22}$ is common. We therefore wished to explore specifically the way in which these patients coped with their role as women, and whether they experienced difficulties with forming relationships. Accordingly, two specific validated measures were used to assess these questions-the Bem sex role inventory $(\mathrm{BSRI})^{24}$ and the inventory of interpersonal problems (IIP). ${ }^{25}$

To assess the level of activity of autonomic innervation, we used a previously validated technique $^{26}$ (laser Doppler flowmetry) to measure rectal mucosal blood flow.

\section{Materials and methods}

SUBJECTS

Thirty seven consecutive female patients (mean age 37 years, range 19-51) referred to a tertiary centre for management of chronic idiopathic constipation were studied. All patients had constipation as defined by the Rome II criteria, ${ }^{27}$ complaining of decreased bowel frequency (less than three times per week), sensation of incomplete emptying or difficult evacuation on at least a quarter of occasions, or a need to strain on at least a quarter of occasions. The mean duration of symptoms was 21 years (range 6-42). Organic and metabolic causes of constipation had been excluded by previous endoscopy, barium enema, and blood testing. All patients had a normal diameter colon. In the week prior to assessment patients were not allowed laxatives. Details were obtained of the patient's bowel frequency, consistency, and need to strain.

Of 37 patients, 21 had three or fewer bowel actions per week, 24 described their stool as hard or pellet-like, 18 needed to strain, and 16 digitated to achieve defecation. All patients complained of either decreased bowel frequency or unsatisfied defecation with an excessive need to strain. This was not quantitated using a diary system; subjective complaints were considered adequate for entry to the study in those patients in whom measured transit was normal. Thirty patients reported some degree of abdominal bloating. Twenty five patients were taking regular laxatives and eight were using suppositories or enemas. Nineteen healthy women (mean age 38 years, range 21-60) with no history of gastrointestinal illness were studied as controls.

Administration of questionnaires, abdominal $x$ ray, and measurement of rectal mucosal blood flow were performed on the same day.

QUESTIONNAIRE ASSESSMENTS

All subjects completed a range of questionnaires in a quiet room on their own. The following instruments were used in the study:

(i) $G H Q-28 .^{18}$ This widely used and reliable measure of psychosocial functioning encompasses four domains-anxiety, depression, somatic symptoms, and social dysfunction.

(ii) "Feminine characteristics" section from the BSRI. ${ }^{24}$ This is a measure of psychological androgyny, namely women's feelings about their femininity. A high score on the BSRI suggests negative feelings about personal femininity. The 15 most "feminine" traits of the inventory were used for the study - the testretest reliability for these 15 items was excellent $(r=0.90)$.

(iii) IIP-subscale of intimacy (IIP-I). ${ }^{25}$ These 12 questions measure an individual's difficulties with forming and maintaining intimate relationships. Eleven of the 12 questions were applied and the test-retest reliability of this modified instrument was also excellent $(r=0.86)$.

\section{COLONIC TRANSIT}

Patients, but not healthy control subjects, had their whole gut transit time assessed using radio-opaque markers (Dunn Clinical Nutrition, Cambridge, UK). These were ingested at 24 hour intervals on three consecutive days and a plain abdominal $x$ ray was obtained 120 hours after ingestion of the first set of markers. ${ }^{28}$ A patient was deemed to have slow transit if there was retention of more than the normal for any one of the three sets of markers. ${ }^{28}$ The remaining patients were classified as having "normal transit constipation" based on their subjective complaints. ${ }^{28}$

RECTAL MUCOSAL BLOOD FLOW STUDIES All subjects underwent laser Doppler flowmetry assessment of rectal mucosal blood flow in a room maintained at $22^{\circ} \mathrm{C}$ after 15 minutes of acclimatisation, in the left lateral position. After digital examination and rigid sigmoidoscopy had confirmed the rectum to be empty, the laser Doppler probe was introduced via the sigmoidoscope. Recordings were made at 10 $\mathrm{cm}$ from the anal verge at four points circumferentially at $90^{\circ}$ to each other, which provides optimum reproducibility. ${ }^{26}$ Readings were taken after the trace had stabilised for 30 seconds. These techniques have been previously validated. ${ }^{26}{ }^{29}$ Measurements were taken during the fasting state and in the follicular phase of the menstrual cycle, these conditions providing a high degree of reproducibility. ${ }^{26}$

STATISTICAL ANALYSIS

Rectal mucosal blood flow data were normally distributed. The two tailed $t$ test was used to compare results of rectal mucosal blood flow 
Table 1 Total general health questionnaire (GHQ) score and subscale scores compared between healthy controls and patients with constipation. Higher scores for each subscale represent greater expression of that characteristic (for example, an anxiety subscale score of 0 represents no expression of anxiety, and a score of 7 represents the highest level of anxiety)

\begin{tabular}{llll}
\hline & $\begin{array}{l}\text { Healthy controls } \\
(n=19)\end{array}$ & $\begin{array}{l}\text { Patients with } \\
\text { constipation }(n=37)\end{array}$ & p Value \\
\hline Total score (0-28) & 5.7 & 12.1 & 0.005 \\
Anxiety subscale (0-7) & 1.9 & 4.1 & 0.001 \\
Depression subscale (0-7) & 0.5 & 2.0 & 0.003 \\
Somatic symptoms subscale (0-7) & 2.2 & 3.2 & 0.005 \\
Social dysfunction subscale (0-7) & 1.2 & 2.8 & 0.003 \\
\hline
\end{tabular}

and questionnaire scores between patients and controls. Spearman's rank order correlation was used to determine associations between the number of retained markers in patients with constipation, mucosal blood flow values, and questionnaire scores. A two tailed $t$ test was used to compare questionnaire scores between slow and normal transit patients. Statistical significance was declared at $\mathrm{p}<0.05$.

The study was given ethics approval by the Northwick Park and St Mark's ethics committee, and informed consent was obtained from all subjects.

\section{Results}

QUESTIONNAIRE ASSESSMENTS

GHQ-28

Compared with healthy controls, women with chronic idiopathic constipation had higher scores on all four subscales of the GHQ-28: anxiety $(p=0.001)$, depression $(p=0.003)$, somatisation $(\mathrm{p}=0.005)$, and social dysfunction $(\mathrm{p}=0.003)$ (table 1). Comparing patients with slow and normal transit, there was no significant difference in three of the four GHQ domains; the somatisation subscale however significantly correlated with whole gut transit $(\mathrm{p}=0.040)$ such that those with slow transit had the highest somatisation scores.

\section{BSRI and IIP-I}

Constipated patients felt less "feminine" and found it harder to form close relationships than healthy women, when assessed using the BSRI $(p=0.05)$ and IIP-I $(p=0.04)$ questionnaires. There were no significant differences in the scores of any of the subscales when comparing patients with slow and normal transit.

\section{WHOLE GUT TRANSIT STUDY}

Fourteen of 37 patients had slow transit. There was no significant difference between slow and normal transit patients when comparing the GHQ total score, BSRI, and IIP. However, there was a significantly $(\mathrm{p}=0.002)$ greater somatisation score on the GHQ-28 subscale in the slow transit patients compared with those with normal transit. Conversely, normal transit patients had significantly greater levels of depression $(p=0.003)$ than slow transit patients.

RECTAL MUCOSAL BLOOD FLOW STUDIES

Rectal mucosal blood flow was reduced in women with constipation compared with healthy controls (151 v 188 flux units; $\mathrm{p}<0.001)$. In constipated patients, there was a significant negative correlation between rectal

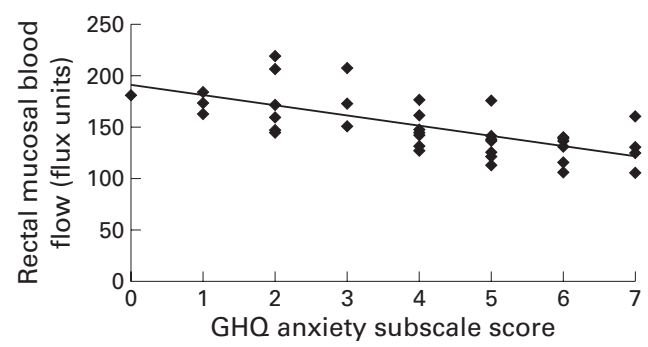

Figure 1 Scattergram showing statistically significant correlation of rectal mucosal blood flow with general health questionnaire (GHO) anxiety subscale in patients with constipation $(r=-0.38, p=0.017)$. High anxiety subscale scores (range 0-7) represent high levels of anxiety.

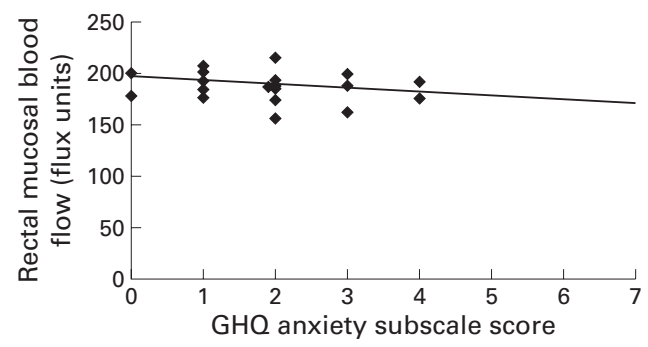

Figure 2 Scattergram showing lack of statistically significant correlation of rectal mucosal blood flow with general health questionnaire (GHQ) anxiety subscale in healthy volunteers $(r=-0.17, p=0.488)$.

mucosal blood flow and the following components of the GHQ-28: somatisation $(r=-0.47$, $\mathrm{p}=0.013)$, anxiety $(r=-0.38, \mathrm{p}=0.017)$ (fig 1$)$, depression $(r=-0.29, \mathrm{p}=0.049)$, social dysfunction $(r=-0.35, \mathrm{p}=0.018)$, and total score $(r=-0.34, \mathrm{p}=0.021)$. There was no significant correlation between any of the GHQ-28 subscales and rectal mucosal blood flow in healthy volunteers (fig 2).

BSRI scores (fig 3) were negatively correlated with mucosal blood flow $(r=-0.50$, $\mathrm{p}=0.0003)$ but IIP score was not $(r=-0.26$, $\mathrm{p}=0.09)$. The higher the psychological morbidity for any scale the lower was rectal mucosal blood flow.

In healthy controls there was no correlation between questionnaire results for any scale or subscale, and mucosal blood flow measurements. This was related, at least in part, to the much narrower range of both psychometric scores and mucosal blood flow in healthy controls.

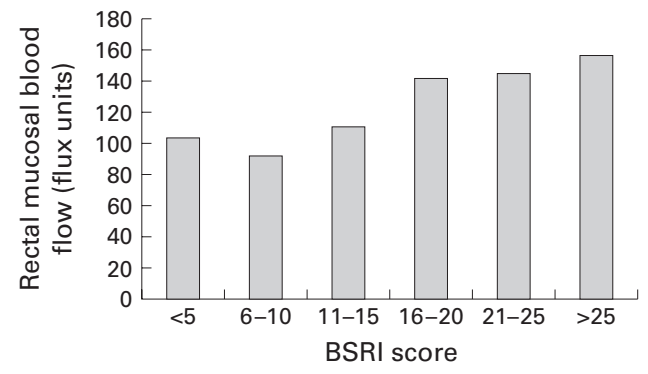

Figure 3 Relationship of measurement of rectal mucosal blood flow with Bem sex role inventory (BSRI) score. The higher the BSRI score the lower the psychological morbidity, and lower flow correlated with poorer psychological score $(r=-0.50, p=0.003)$. 


\section{Discussion}

This study has demonstrated that women with idiopathic constipation have significantly greater psychological morbidity than age matched healthy controls. In particular, in this group of women who presented to a tertiary referral centre, they had greater levels of anxiety, depression, somatisation, and social dysfunction. Previous studies on similar patient groups have shown that patients who are constipated and have normal transit have higher levels of depression than those with slow transit. ${ }^{15} 16$ This finding was reproduced in our subjects. Additionally, those with normal transit had lower levels of somatisation than those with slow transit. This raises the possibility of a link between somatisation and altered gut innervation, reflecting a possible efferent cholinergic underactivity and/or sympathetic overactivity. Support for these abnormalities comes from previous studies of peripheral autonomic testing in these patients. ${ }^{12} 132930$

Demonstration that patients in this study had abnormal psychological measures compared with controls reflects the previously documented underlying tendency towards depression and anxiety in this patient group. ${ }^{15}{ }^{16} \mathrm{It}$ is known that patients with depression have lower resting vagal tone, ${ }^{31}$ and that patients with anxiety have higher sympathetic tone $\mathrm{e}^{32}$ and higher parasympathetic tone. ${ }^{33}$ Alterations in mucosal blood flow seen in patients with constipation $^{29}$ and the correlation with the abnormal psychological measures found in constipated patients demonstrated in this study support the hypothesis that the extrinsic autonomic innervation to the gut is disturbed in these patients with constipation. This effect would seem to be most marked in slow transit constipation.

Patients in this study did not have bowel function validated by diary card. However, transit time was formally assessed in all patients. Those with normal transit still expressed the subjective feeling of unsatisfied defecation, this being the basis on which this group with normal transit was defined for the purpose of this study. Patient reported symptoms have been shown to be correlated with a diary card record in our institution. ${ }^{34}$

As shown in a previous study, ${ }^{29}$ we have demonstrated that rectal mucosal blood flow is reduced in patients with idiopathic constipation. The main determinant of extrinsic control of the gut mucosal microcirculation is thought to be the sympathetic system which provides a predominantly inhibitory tone to the small vessels. ${ }^{35}$ Furthermore, intestinal blood vessels are cholinergically innervated ${ }^{36-39}$ and there is a balanced sympathovagal activation of vasoconstriction and vasodilatation, respectively. ${ }^{4041}$ The greater the score on psychometric testing, especially in the GHQ somatisation and anxiety subscales, the lower the mucosal blood flow reading, suggesting a direct link between chronic psychological state and gut function. Anxiety is known to be associated with increased sympathetic outflow, ${ }^{41}$ traditionally measured via altered cardiovascular parameters, but this study provides direct evidence that there is also a more specific direct measurable effect via the gut extrinsic innervation. One previous study in constipated patients demonstrated a relationship between anxiety and altered transit in the ascending colon, with slower transit in those with greater anxiety. ${ }^{42}$ The sympathetic system is known to have an inhibitory effect on gut transit, ${ }^{43}$ providing complementary evidence for an effect of this altered innervation. It is possible that altered autonomic outflow, in particular increased sympathetic and diminished parasympathetic drive, due to chronic central changes in psychological state, result in decreased motility. However, a reduction in blood flow related to diminished intrinsic neural activity cannot be completely excluded.

The changes demonstrated in rectal mucosal blood flow may parallel these motility changes as a direct result of the same changes in autonomic activity. Changes in blood flow should not be thought of as secondary to changes in motility; changes in motility, blood flow, and secretion are likely to occur simultaneously as is seen in acute experiments. ${ }^{9-11}$ However, a change in mucosal blood flow secondary to altered motor function cannot be excluded. In this regard it is of note that there were several correlations between psychological measures and mucosal blood flow. There were also correlations between psychological measures and whole gut transit. This raises the possibility of function specific control of gut function by motility regulating, blood flow regulating, and secretomotor neurones. Future studies powered to look at this phenomenon of function specific regulation of gut function may be revealing.

The results of this study complement other recent studies which have used new imaging techniques to show that patients with functional bowel disorders have altered levels of cerebral arousal. ${ }^{44}{ }^{45}$ This study extends these observations by demonstrating the mechanism by which these cerebral changes influence gut function in humans. In a previous study, ${ }^{46} \mathrm{a}$ group of constipated patients who had a successful response to biofeedback therapy were shown to exhibit an associated improvement in rectal mucosal blood flow. This also correlated with improved psychological state. There was no change with treatment in non-gut measures of autonomic function, such as altered cardiovascular reflexes, suggesting that this technique not only demonstrates altered innervation but is also sensitive to changes with treatment. That study also provided evidence that altered mucosal blood flow is related to changeable extrinsic neurological control, rather than permanent damage to intrinsic nerves.

Other possible causes for altered mucosal blood flow need to be considered. This blood flow alteration may be an association of the change in large bowel motility, although its reduction in patients with normal transit constipation would not support this. Another possibility is that altered circulating hormones associated with anxiety, such as catecholamines, have an effect on the mucosal 
microcirculation. In the first study it was shown that acute stimulation of sacral nerves immediately alters mucosal blood flow, confirming the effect of extrinsic nerves. ${ }^{26}$ However, an additional circulating hormonal effect cannot be excluded.

This study has provided quantitative evidence for a direct relationship between measured chronic psychological morbidity and altered extrinsic innervation to the gut. Further studies may reveal the mechanism by which different treatments of functional disorders cause change in psychological state and level of activity of gut innervation.

This work has been published as an abstract (Emmanuel AV, Kamm MA. Gastroenterology 1997;112 (suppl):A728).

1 Sakaguchi $T$, Ohtake $M$. Inhibition of gastric motility induced by activation of the hypothalmic paraventricular nucleus. Brain Res 1985;335:365-9.

2 Fengs HS, Brobeck JR, Brooks FP. Lateral hypothalamic sites in cats for stimulation of gastric and antral sites in cats for stimulation of gastric
contractions. Clin Invest Med 1987;10:140-5.

3 Smith JR, LaHann TR, Chesnut RM, et al. Thyrotropinreleasing hormone: stimulation of colonic activity following intracerebroventricular administration. Science 1977;196: 660-1

4 Bueno L, Ferre JP. Central regulation of intestinal motility by somatostatin and cholecystokinin octapeptide. Science 1982;216:1427-9.

5 Tache Y, Garrick T, Raybould H. Central nervous system: action of peptides to influence gastrointestinal motor function. Gastroenterology 1990;98:517-28.

6 Bueno L, Gue M. Evidence for the involvement of corticotropin-releasing factor in the gastrointestinal disturbances induced by acoustic and cold stress in mice. Brain Res 1988;441:1-4.

7 Gray JA. The neuropsychology of anxiety. Oxford: Clarendon Press, 1974:16-31.

8 O'Brien JT. The "glucocorticoid cascade" hypothesis in man. Br f Psychiatry 1997;170:199-201.

9 Beaumont W. Experiments and observations on the gastric juice and the pathophysiology of digestion. Plattsburg, New York: and the pathophysic

10 Wolf S, Wolf HG. Experimental study of changes in gastric function in response to varying life experiences. Rev Gastroenterol 1947;14:419-26.

11 Almy TP. Experimental studies on the irritable colon. $A m \mathcal{F}$ Med 1951;10:60-7.

12 Aggarwal A, Cutts TF, Abell TL, et al. Predominant symptoms in irritable bowel syndrome correlate with specific autonomic nervous system abnormalities. Gastroenterology 1994;106:945-50.

13 Altomare D, Pilot MA, Scott M, et al. Detection of a subclinical autonomic neuropathy in constipated patients using a sweat test. Gut 1992;33:1539-43.

14 Wald A, Hinds JP, Caruana BJ. Psychological and physiological characteristics of patients with severe idiophysiological characteristics of patients with sever

15 Devroede G, Girard G, Bouchoucha M, et al. Idiopathic constipation by colonic constipation by colonic
dysfunction: relationship with personality and anxiety. Dig dysfunction: relationship
Dis Sci 1989;34:1428-33.

16 Preston DM, Pfeffer J, Lennard-Jones JE. Psychiatric assessment of patients with severe constipation. Gut 1984; 25:A582-3

17 Mason HJ, Kamm MA, Serrano-Ikkos I. Women with idiopathic constipation have marked psychological morbidity, altered female self perception and increased somatisation compared with healthy women and women with Crohn's disease. Gastroenterology 1997;112:A784.

18 Preston DM, Lennard-Jones JE. Severe chronic constipation of young women: "idiopathic slow transit constipation". Gut 1986;27:41-8.

19 Drossman DA, Leserman J, Nachman G, et al. Sexual and physical abuse in women with functional or organic gastrointestinal disorders. Ann Intern Med 1990;113:828-33.
20 Leroi AM, Bernier C, Watier A, et al. Prevalence of sexual abuse among patients with functional disorders of the lower gastrointestinal tract. Int $\mathcal{F}$ Colorectal Dis 1995;10:200-6.

21 Bem SL. The measurement of psychological androgyny. $f$ Consult Clin Psychol 1974;42:155-62.

22 Horowitz LM, Rosenberg SE, Baer BA, et al. Inventory of interpersonal problems: psychometric properties and clinical applications. F Consult Clin Psychol 1988;56:885-96.

23 Goldberg DP. Manual of the general health questionnaire (GHQ-28). Toronto: NFER Nelson Publishing, 1981.

24 Lykouras L, Adrachta D, Kalfakis N, et al. GHQ-28 as an aid to detect mental disorders in neurological in-patients. Acta Psychiatr Scand 1996;93:212-16.

25 Banks MH. Validation of the general health questionnaire in a young community sample. Psychol Med 1983;13:349-53.

26 Emmanuel AV, Kamm MA. Emmanuel AV, et al. Laser Doppler measurement of mucosal blood flow. Gut 1999;45:64-9.

27 Thompson WG, Longstreth GF, Drossman DA, et al. Functional bowel disorders and functional abdominal pain. Gut 1999;45(suppl II):I143-7.

28 Evans RC, Kamm MA, Hinton JM, et al. The normal range and a simple diagram for recording whole gut transit time. Int $\mathcal{F}$ Colorectal Dis 1992;7:15-17.

29 Emmanuel AV, Kamm MA. Laser Doppler flowmetry as a measure of extrinsic colonic innervation in functional bowel disease. Gut 2000;46:212-17.

30 Jorgensen LS, Christiansen P, Raundahl U, et al. Autonomic nervous system function in patients with functional abdominal pain. An experimental study. Scand f Gastroenterol 1993;28:63-8.

31 Krittayaphong R, Cascio WE, Light $\mathrm{KC}$, et al. Heart rate variability in patients with coronary artery disease: differences in patients with higher and lower depression scores. Psychosom Med 1997;59:231-5.

32 Piccirillo G, Viola E, Nocco $M$, et al. Autonomic modulation and QT interval dispersion in hypertensive subjects with anxiety. Hypertension 1999;34:242-6.

33 Punyabati O, Deepak KK, Sharma MP, et al. Autonomic nervous system reactivity in irritable bowel syndrome. Indian $\mathcal{F}$ Gastroenterol 2000;19:122-5.

34 Emmanuel AV, Nicholls T, Roy AJ, et al. Prucalopride improves colonic transit and stool frequency in patients with slow and normal transit constipation. Gastroenterology 2000;118(supp 2):A4438.

35 Burnstock G. Integration of factors controlling vascular tone-an overview. Anesthesiology 1993;79:1368-80.

36 Thiefin G, Raybould HE, Leung FW, et al. Capsaicinsensitive afferent fibres contribute to gastric mucosal blood flow response to electrical vagal stimulation. Am $\mathcal{F}$ Physiol 1990;259:G1037-43.

37 Beyak M, Vanner S. Histamine $\mathrm{H}_{1}$ and $\mathrm{H}_{3}$ vasodilator mechanisms in the guinea pig ileum. Gastroenterology 1995; 108:712-18

38 Andriantsitohaina N, Suprenant A. Acetylcholine released from guinea-pig submucosal neurones dilates arterioles by releasing nitric oxide from endothelium. F Physiol 1992; 453:493-502.

39 Thiefin G, Leung FW, Tache Y, et al. Dissociated effects of corticotropin-releasing factor on acid secretion and blood flow. Am f Physiol 1989;256:G412-17.

40 Vanner S, Suprenant A. Neural reflexes controlling intestinal microcirculation. Am f Physiol 1996;271:G223-30.

41 Chaudhuri KR, Thomaides T, Mathias CJ. Abnormality of superior mesenteric blood flow in human sympathetic failure. F Physiol (Lond) 1992;457:477-89.

42 Devroede G. A clinical perspective of psychological factors in constipation. In: Kamm MA, Lennard-Jones JE, eds. Constipation. Petersfield, UK: Wrightson Biomedical Publishing, 1994

43 McIntyre AS, Thompson DG. Review article: adrenergic control of motor and secretory function in the gastrointestinal tract. Aliment Pharmacol Ther 1992;6:125-42.

44 Creed FH, Guthrie E. Progress report: psychological factors in the irritable bowel syndrome. Gut 1987;28:1307-18.

45 Mayer EA, Gebhart GF. Basic and clinical aspects of visceral hyperalgesia. Gastroenterology 1995;107:271-93

46 Emmanuel AV, Kamm MA. Successful response to biofeedback for constipation is associated with specifically improved extrinsic autonomic innervation to the large bowel. Gastroenterology 1997;112(suppl):A729. 